\author{
Niyazova R..$^{*}$, Atambayeva Sh. ${ }^{1}$, Akimniyazova A. ${ }^{1}$, Pinsky I. ${ }^{1}$, \\ Alybaeva A. ${ }^{1}$, Faye B. ${ }^{2}$, Ivashchenko A. ${ }^{1}$ \\ ${ }^{1}$ al-Farabi Kazakh National University, Almaty, Kazakhstan \\ ${ }^{2}$ CIRAD, Montpellier, France \\ "e-mail: raiguln@mail.ru
}

\title{
Features of mir-466-3p binding sites in mRNA genes with different functions
}

\begin{abstract}
The importance of miRNA in cellular regulation is gaining momentum. We searched miRNA binding sites using the MirTarget program. We identified several miRNAs that have greater than 300 target genes in humans, with multiple binding sites per gene. We observed that miR-466-3p 1,463 binding sites with high affinity (with $\Delta \mathrm{G} / \Delta \mathrm{G}_{\mathrm{m}}$ values greater than or equal to $90 \%$ ).
\end{abstract}

Key words: binding, sites, genes, functions.

\section{Introduction}

Numerous microRNAs (miRNAs) that regulate the expression of several hundred genes by binding to their mRNAs have been identified [1-4]. These miRNAs play key roles in the regulation of many biological processes and changes in their concentrations are observed in various pathologies in humans and other animals [5-7]. Changes in miRNA concentrations can be a cause or a consequence of a disease.

\section{Materials and methods}

The human gene mRNAs were taken from the GenBank (http://www.ncbi.nlm.nih.gov) using Lextractor002 script (http://sites.google.com/site/malaheenee/software). Human miR-466-3p was taken from the miRBase site (http://mirbase.org).

The target genes for the tested miRNA were revealed using the MirTarget program, which was developed in our laboratory. This program defines the following features of binding: a) the beginning of an miRNA binding with mRNAs; b) the localization of miRNA binding sites in the 5'-untranslated regions (5'UTRs), coding domain sequences (CDSs) and 3'-untranslated regions (3'UTRs) of the mRNAs; c) the free energy of hybridization $(\Delta \mathrm{G}, \mathrm{kJ} / \mathrm{mole})$; and d) the schemes of nucleotide interactions between the miRNAs and the mRNAs. The ratio $\Delta \mathrm{G} / \Delta \mathrm{G}_{\mathrm{m}}$ (\%) was counted for each site, where $\Delta \mathrm{G}_{\mathrm{m}}$ equaled the free energy of a miRNA binding with its perfect complementary nucleotide sequence. The miRNA binding sites located on the mRNAs had $\Delta \mathrm{G} / \Delta \mathrm{G}_{\mathrm{m}}$ ratios of $90 \%$ and more. We also note the position of the binding sites on the mRNA, beginning from the first nucleotide of the mRNA's 5'UTR. The MirTarget program computes the interactions between the nucleotides of miRNAs and those of target gene mRNAs. It found bonds between adenine (A) and uracil (U), guanine $(\mathrm{G})$ and cytosine $(\mathrm{C})$, and $\mathrm{G}$ and $\mathrm{U}$, as well as between $\mathrm{A}$ and $\mathrm{C}$ via a hydrogen bond [8]. The distance between $\mathrm{A}$ and $\mathrm{C}$ was equal to the $\mathrm{G}-\mathrm{C}, \mathrm{A}-\mathrm{U}$, and $\mathrm{G}-\mathrm{U}$ distances [9]. The numbers of hydrogen bonds in the G-C, A-U, G-U and A-C interactions were found to be $3,2,1$ and 1 , respectively. The free binding energies of these nucleotide pairs were accepted as the same values $(3: 2: 1: 1)$.

\section{Results and their discussion}

One miRNA that has multiple binding sites is miR-466-3p, which has more than 300 target genes. Their mRNAs include a total of 1,463 binding sites, including from 1 to 18 sites in RAPGEFL1 and SYT2, 25 in $P R K X$, and 38 sites in DONSON mRNA. The miRNA binding sites have $\Delta \mathrm{G} / \Delta \mathrm{G}_{\mathrm{m}}$ ratios of $90 \%$ or greater. This indicates a high degree of interaction between miRNAs and mRNAs. Multiple binding sites increase the probability that miRNAs interact with mRNAs, and as a consequence, the translation of such mRNAs is reduced.

The target genes of miR-466-3p serve various functions. Many are transcription factors and kinases, some are involved in the cell cycle, apoptosis, and other processes (Table «a»). 
Table «a» - The characteristics of miR-466-3p binding in the 5'UTR of mRNA target genes

\begin{tabular}{|c|c|c|c|c|c|}
\hline Gene & $\begin{array}{c}\text { Start positions of } \\
\text { binding sites, } \mathrm{nt}\end{array}$ & $\Delta \mathrm{G}, \mathrm{kJ} / \mathrm{mole}$ & Gene & $\begin{array}{c}\text { Start positions of } \\
\text { binding sites, } \mathrm{nt}\end{array}$ & $\Delta \mathrm{G}, \mathrm{kJ} / \mathrm{mole}$ \\
\hline BHLHE41 & $89(1)^{*}$ & -106.1 & $R U N D C 3 B$ & $172-160(10)$ & $-106.1 \div-106.2$ \\
\hline CASQ2 & $31(1)$ & -106.1 & $S P O P$ & $79(1)$ & -106.2 \\
\hline CD97 & $140-138(11)$ & $-106.2 \div 108.3$ & STAT6 & $102-100(5)$ & $-106.2 \div 108.3$ \\
\hline CELF4 & $299(1)$ & -108.3 & TSHZ2 & $22(1)$ & -106.2 \\
\hline CHRNA2 & $9-23(3)$ & $-106.2 \div 108.3$ & VWA3A & $17-23(2)$ & $-108.3 \div 110.4$ \\
\hline GRIA2 & $52-68(3)$ & -106.2 & XPO1 & $499-507(5)$ & -106.2 \\
\hline KIAA0319 & $265-291(14)$ & -106.2 & ZNF331 & $409-415(4)$ & -106.2 \\
\hline NOS1AP & $2099(1)$ & -106.2 & & \\
\hline \multicolumn{7}{r}{ Note. ${ }^{*}$ In brackets are number of binding sites } \\
\hline
\end{tabular}

One of the objectives of this work was to identify the target genes of miR-466-3p, which may contribute to the development of several diseases. The mRNA of ADAT2, ADCYAP1R1, BHLHE40, CDK6, FASLG, FGFR3, FLT1, MACC1, MAPKAPK2, MECOM, SPN, STAT6, and UGT8 genes, which are involved in the development of lung and breast cancer, are associated with miR-466-3p, indicating an increased probability that miR-466-3p influences these types of cancer. $A D R B K 2, B H L$ HE40, CD36, EGR3, NDUFS2, NFAT5, NOS1, PLA2G7, S1PR2, and STAT6 genes are responsible for diseases of the cardiovascular system, and their mRNAs also interact with miR-466-3p. CDK6 and $M E C O M$ genes are involved in cell cycle regulation and apoptosis, and their mRNA sequences contain targets for miR-466-3p. We identified the $B H L$ $H E 40$ gene, which exhibits changes in expression in lung cancer, breast cancer, cardiovascular diseases, and circadian rhythm disorders. BHLHE40 is a transcription factor that is expressed in various tissue types. It encodes a protein involved in the control of circadian rhythms, cell differentiation, proliferation, cell cycle, apoptosis, and the development of various diseases. Therefore, a change in the expression of this gene after binding with miR-466-3p can cause many diseases.

In addition to mir-466, we identified several miRNAs that have greater than 300 target genes in humans, with multiple binding sites per gene. We observed that miR-3960 has 2,563 mRNA binding sites with high affinity for 375 human genes. For example, miR-3960 has 565 binding sites in 5' UTRs and 515 sites in mRNA coding sequences (CDS). The mRNA regions that contain several miR-3960 binding sites have starting sites located through one, two, or three nucleotides. The nucleotide sequences of the binding sites located in CDSs encode polyalanine or polyproline. We determined that most of the target genes for the miRNAs examined encode transcription factors. Specifically, miR-3960, miR-3620-5p, and miR-8072 bind with genes involved in cell cycle regulation and apoptosis. Hsa-miR-1322 has more than 2,000 binding sites in the mRNAs of 1,058 genes. This includes 1,889 binding sites in CDSs, 215 binding sites in 5', UTRs, and 160 binding sites in 3' UTRs. Between 2 and 28 binding sites were arranged sequentially with start positions overlapping with three nucleotides of the adjacent binding site. The nucleotide sequences of these sites in CDSs encode oligopeptides with the same and/or different amino acid sequences. We found that $33 \%$ of the target genes encoded transcription factors. The miR-1322 binding sites has arranged binding sites were arranged in the CDSs of the orthologous genes MAMLD1, MAML2, and $M A M L 3$ genes. These sites encode a polyglutamine oligopeptide ranging from six 6 to 47 amino acids in length $[1,10]$.

We identified 266 target human genes for miR574-5p and six target genes for miR-574-3p. The miR-574-5p binding sites were mainly located mainly in the 3' UTRs, and theirthere number is equal towere 1,429 in total. The miR-574-5p binding sites were located in the 3' UTRs of the mRNA sequences of 244 genes mRNAs, in the 5' UTRs of 20 genes mRNAs and in the CDSs of two genes mRNAs. The miR-574-5p binding sites in the CDS of FGFRL1 and REM2 genes mRNAs encode the oligopeptides HTHTHTHS and DTDMDTDT in the relevant proteins. The beginning start sites of multiple miR-574$5 \mathrm{p}$ binding sites arranged located through two nucleotides and the number of binding sites in one region 
ranged from 1 to 37 . The target genes of miR-574-5p have been implicated in the development of breast and, lung cancer and other diseases. A significant substantial part portion of the target genes of miR574-5p are transcription factors and kinases, which are involved in apoptosis and the cell cycle. The synthesis of miR-574-5p and miR-574-3p depends on the expression of the master's gene FAM114A1 expression, which is the target for of 15 miRNAs. Changes in the expression of miR-574-5p and miR574-3p are correlated with changes in the expression of their target genes, at which are associated with the development of many pathologies, including cardiovascular diseases and cancer [11].
Table «a» shows the characteristics of miR-466-3p binding sites in the 5' UTRs of 15 target genes mRNAs with the value of $\Delta \mathrm{G} / \Delta \mathrm{Gm}$ values that were equal or greater than $90 \%$. The number of miR-466-3p binding sites with mRNAs offor these genes varied from one 1 to 14 (Table $\langle\mathrm{b} »,\langle\mathrm{c}\rangle)$, indicating the variation in the probability of an interaction between miR-466-3p interaction and target mRNAs, since the greater the length of the section of longer binding sites, is associated with a higher the probability of interaction. It is possible that the mRNA of the KIAA0319 gene may contacts with two RISC complexes containing miR-466-3p, since the length of the site from nucleotides 265 to $314 \mathrm{nt}$, which contains multiple binding sites, is $50 \mathrm{nt}$.

Table «b» - The characteristics of miR-466-3p binding in the 3'UTR of mRNA target genes having 1-4 sites

\begin{tabular}{|c|c|c|c|c|c|}
\hline Gene & $\begin{array}{l}\text { Start positions of } \\
\text { binding sites, nt }\end{array}$ & $\Delta \mathrm{G}, \mathrm{kJ} / \mathrm{mole}$ & Gene & $\begin{array}{l}\text { Start positions of } \\
\text { binding sites, nt }\end{array}$ & $\Delta \mathrm{G}, \mathrm{kJ} / \mathrm{mole}$ \\
\hline ADAMTS4 & $3382(1)$ & -110.4 & LSAMP & $2285(1)$ & -106.2 \\
\hline$A D R B 3$ & $2451(1)$ & -110.4 & MAPKAPK2 & $2587-2589(2)$ & $-106.2 \div-108.3$ \\
\hline AKIRIN1 & 1057 (1) & -106.2 & MECOM & $5099(1)$ & -110.4 \\
\hline$A Q P 3$ & $1254-1477(2)$ & -106.2 & MED24 & $3606-3612(4)$ & $-106.2 \div-108.3$ \\
\hline ARHGAP26 & $8270(1)$ & -106.2 & METTL16 & $3581-3585(4)$ & $-106.2 \div-108.3$ \\
\hline$A R N T$ & 3104 (1) & -106.2 & $M V B 12 B$ & $3440-3438(2)$ & $-106.2 \div-108.3$ \\
\hline ATAD1 & $1830(1)$ & -106.2 & MYO16 & $6426(1)$ & -106.2 \\
\hline ATG10 & $1928(1)$ & -108.3 & $N A C C 2$ & $2484-5875(2)$ & $-106.1 \div-108.3$ \\
\hline ATP6V0D1 & $1201-1211(3)$ & $-106.1 \div 110.4$ & NAV1 & $9412-9418(4)$ & -106.2 \\
\hline$A T X N 7 L 3 B$ & $3359(1)$ & -108.3 & NCOA3 & $5966-5974(2)$ & $-106.2 \div-108.3$ \\
\hline BAHCC1 & $8118(1)$ & -108.3 & NET1 & $2642(1)$ & -106.2 \\
\hline BHLHE40 & $1687-1693(4)$ & $-106.2 \div-108.3$ & NFAT5 & $8540-8548(3)$ & $-106.2 \div-108.3$ \\
\hline$B N C 2$ & $3528-3534(3)$ & -106.2 & NPAP1 & $5206(1)$ & -106.2 \\
\hline$B S N$ & $15555(1)$ & -106.1 & NPTXR & $5706-5712(3)$ & $-106.2 \div-108.3$ \\
\hline C16orf52 & $1410-1416(2)$ & $-108.3 \div-110.4$ & NR1D2 & $3337(1)$ & -110.4 \\
\hline C19orf59 & $816(1)$ & -106.1 & $O C L N$ & $3570-3572(2)$ & $-106.2 \div-108.3$ \\
\hline C3orf72 & $2537-2543(2)$ & -108.3 & PARD3B & $4736(1)$ & -110.4 \\
\hline C9orf47 & $1531(1)$ & -106.2 & PDE7A & $2666(1)$ & -106.2 \\
\hline$C A C N A 1 B$ & $8725-8731(4)$ & $-106.2 \div-108.3$ & PHKA1 & $5211-5213(4)$ & $-106.2 \div-108.3$ \\
\hline CADM3 & $2634-2646(2)$ & -106.2 & PIK3R1 & 6519-6523 (4) & $-106.2 \div-108.3$ \\
\hline CD19 & $1861-1863(2)$ & $-106.2 \div-108.3$ & PLAG1 & $4546(1)$ & -106.2 \\
\hline CD3EAP & 3086-3092 (4) & -106.2 & PLCXD1 & $2548(1)$ & -108.3 \\
\hline CEP135 & $5219-5221(2)$ & $-106.2 \div-108.3$ & PPARGCIA & $2806-2822(2)$ & -106.2 \\
\hline CEP85L & $3355(1)$ & -106.2 & PPARGCIB & $9983(1)$ & -106.2 \\
\hline CISD2 & 1146-1144 (4) & $-106.2 \div-108.3$ & PPIC & 781-787 (4) & -106.2 \\
\hline CLEC4D & $1001(1)$ & -108.3 & PRR5L & $3504(1)$ & -106.2 \\
\hline CLN8 & $2553-2555(2)$ & -106.2 & PRSS23 & $1454-1458(3)$ & -106.2 \\
\hline CLRN1 & $1303-1309$ (4) & $-106.2 \div-108.3$ & PSD3 & $4157-4161(2)$ & $-106.2 \div-110.4$ \\
\hline
\end{tabular}


Continuation of Table «b»

\begin{tabular}{|c|c|c|c|c|c|}
\hline Gene & $\begin{array}{l}\text { Start positions of } \\
\text { binding sites, nt }\end{array}$ & $\Delta \mathrm{G}, \mathrm{kJ} / \mathrm{mole}$ & Gene & $\begin{array}{l}\text { Start positions of } \\
\text { binding sites, nt }\end{array}$ & $\Delta \mathrm{G}, \mathrm{kJ} / \mathrm{mole}$ \\
\hline$D D B 1$ & $3990-3998(3)$ & $-106.2 \div-108.3$ & PTCH1 & $6512-6520(3)$ & -106.1 \\
\hline$D G K G$ & $3890-3908$ (4) & $-106.1 \div-106.2$ & RNF121 & $2208-2214(3)$ & -106.2 \\
\hline DOK6 & $1761(1)$ & -110.4 & $R U N X 1$ & $5455-5459(2)$ & $-106.2 \div-110.4$ \\
\hline DPYSL5 & $4857(1)$ & -106.1 & S1PR2 & $3189(1)$ & -110.4 \\
\hline DTNA & $5953(1)$ & -106.2 & $S B K 1$ & $4530(1)$ & -110.4 \\
\hline DYNC1LI1 & $1796(2)$ & -106.2 & SEL1L3 & $4370-4374(3)$ & $-106.2 \div-108.3$ \\
\hline EGR3 & $2026(1)$ & -108.3 & SGPL1 & $3972-3974(2)$ & $-106.2 \div-108.3$ \\
\hline EPG5 & $8002(1)$ & -106.2 & SIK1 & $2855-2857(2)$ & -106.2 \\
\hline EPHB3 & $4065(1)$ & -106.2 & $S L C 13 A 2$ & $2189(1)$ & -106.2 \\
\hline$F 11 R$ & $4268(1)$ & -106.2 & SLC16A9 & $2962(1)$ & -106.2 \\
\hline FAM105B & $5211(1)$ & -106.2 & SLC1A4 & $4073(1)$ & -108.3 \\
\hline FAM178A & $4992-4996(3)$ & $-106.2 \div-108.3$ & $S L C 25 A 22$ & $2213(1)$ & -106.2 \\
\hline FAM46C & $5184(1)$ & -106.1 & SLC35E3 & $1905(1)$ & -106.2 \\
\hline FBXO9 & $1718-1720(2)$ & $-106.2 \div-112.5$ & SLC6A6 & $2306-2314(2)$ & $-106.2 \div-110.4$ \\
\hline FGFR3 & $2809(1)$ & -110.4 & SLC7A11 & $3438-3446(3)$ & $-106.2 \div-108.3$ \\
\hline FHOD1 & $3751-3759(2)$ & -106.2 & $S O G A 3$ & $8790(1)$ & -106.2 \\
\hline FLVCR2 & $3355(1)$ & -106.2 & $S P N$ & $4373-4387$ (2) & $-106.2 \div-108.3$ \\
\hline FOXI2 & $2790(1)$ & -106.1 & SPOCK 2 & $1917(1)$ & -108.3 \\
\hline FRMD3 & 3217 & -108.3 & STARD 8 & $4681(1)$ & -110.4 \\
\hline FXYD6 & 963-1138 (4) & $-106.2 \div-108.3$ & STAT5B & $2717-2721(3)$ & -106.2 \\
\hline GADL1 & $3346(1)$ & -106.2 & STRBP & $6071-6073(2)$ & -106.2 \\
\hline GOLGA7B & $5601(1)$ & -106.2 & $S T X 1 B$ & $1032-1180(4)$ & $-106.2 \div-108.3$ \\
\hline$G P X 3$ & $1108(1)$ & -106.2 & TFCP2 & $3457-3610(3)$ & -106.2 \\
\hline GRID1 & $5455(1)$ & -106.2 & TM9SF3 & $4448(1)$ & -106.2 \\
\hline GRIK4 & 4357 (1) & -106.1 & $T M C 7$ & $4230(1)$ & -106.2 \\
\hline GSK3B & $4673(1)$ & -106.2 & TMOD2 & $8207(1)$ & -108.3 \\
\hline$H I C 2$ & $3104-3122(2)$ & $-106.2 \div-108.3$ & UGT8 & $2500-2516(3)$ & $-106.2 \div-108.3$ \\
\hline HIF $1 A N$ & $2037-2041(2)$ & -108.3 & UNC80 & $11147(1)$ & -110.4 \\
\hline$H L F$ & $5348-1554(3)$ & $-106.2 \div-110.4$ & USHIG & $2580-2745(3)$ & -106.2 \\
\hline ICAMI & $2988(1)$ & -106.2 & VPS13D & 14819-14821(2) & -106.2 \\
\hline$I G F B P 4$ & $1230-1234(2)$ & -106.1 & WDR35 & $6259(1)$ & -106.2 \\
\hline$I M P G 2$ & $5471(1)$ & -114.6 & WDR37 & $4026-4030(3)$ & -106.2 \\
\hline IRS1 & $7318-7320(2)$ & $-106.2 \div-108.3$ & $W D R 5 B$ & $2049(1)$ & -106.2 \\
\hline ITGAM & $3749(1)$ & -106.1 & WDR72 & 5941 & -106.2 \\
\hline KCTD11 & $2640-2644(3)$ & -106.2 & WSCD2 & $4274-4276(4)$ & $-106.2 \div-108.3$ \\
\hline KCTD16 & $3932(1)$ & -108.3 & ZBTB42 & 3075-3077 (3) & $-106.2 \div-108.3$ \\
\hline KIAA0408 & $5096(1)$ & -106.2 & ZDHHC22 & $1832-1836(3)$ & -106.2 \\
\hline KIAA1804 & $5327(1)$ & -108.3 & ZEB2 & $6533(1)$ & -106.2 \\
\hline KIAA2022 & $5693(1)$ & -106.2 & ZNF33A & $3169(1)$ & -108.3 \\
\hline KIF13A & $6965(1)$ & -106.2 & ZNF33B & $3103(1)$ & -106.2 \\
\hline KIF3C & $3122(1)$ & -106.1 & ZNF346 & $2186-2202(2)$ & -108.3 \\
\hline KLHL3 & $2677-2679(2)$ & -106.2 & ZNF428 & $1214-1224(2)$ & $-106.2 \div-108.3$ \\
\hline
\end{tabular}


Continuation of Table «b»

\begin{tabular}{|c|c|c|c|c|c|}
\hline Gene & $\begin{array}{c}\text { Start positions of } \\
\text { binding sites, } \mathrm{nt}\end{array}$ & $\Delta \mathrm{G}, \mathrm{kJ} / \mathrm{mole}$ & Gene & $\begin{array}{c}\text { Start positions of } \\
\text { binding sites, } \mathrm{nt}\end{array}$ & $\Delta \mathrm{G}, \mathrm{kJ} / \mathrm{mole}$ \\
\hline KLHL42 & $3780(1)$ & -108.3 & ZNF483 & $3636-3638(3)$ & $-106.2 \div-108.3$ \\
\hline LFNG & $1270(1)$ & -108.3 & ZNF562 & $3963-3971(3)$ & $-106.2 \div-110.4$ \\
\hline LILRB2 & $2858-2864(4)$ & $-106.2 \div-108.3$ & ZNF618 & $2835(1)$ & -106.2 \\
\hline LONRF1 & $2685(1)$ & -106.1 & & & \\
\hline
\end{tabular}

Table $« \mathbf{c} »-$ The characteristics of miR-466-3p binding in the 3'UTR of mRNA target genes having five and more binding sites

\begin{tabular}{|c|c|c|c|c|c|}
\hline Gene & $\begin{array}{l}\text { Start positions of } \\
\text { binding sites, nt }\end{array}$ & $\Delta \mathrm{G} / \Delta \mathrm{G}_{\mathrm{m}}, \mathrm{kJ} / \mathrm{mole}$ & Gene & $\begin{array}{l}\text { Start positions of } \\
\text { binding sites, nt }\end{array}$ & $\Delta \mathrm{G} / \Delta \mathrm{G}_{\mathrm{m}}, \mathrm{kJ} / \mathrm{mole}$ \\
\hline ABLIM1 & $4472-4498$ (14) & -106.2 & MLLT6 & $4051-4075(6)$ & $-106.2 \div-110.4$ \\
\hline$A D A T 2$ & $1950-1964(8)$ & -106.2 & $M M S 22 L$ & $5738-5754(9)$ & -106.2 \\
\hline$A D C Y A P 1 R 1$ & $3841-3853(7)$ & $-106.2 \div-108.3$ & $M Y A D M$ & 1982-1992(6) & -106.2 \\
\hline$A D R B K 2$ & 6676- $6696(11)$ & -106.2 & MYSM1 & $3512-3524(7)$ & -106.2 \\
\hline$A F F 1$ & $6829-6837(5)$ & -106.2 & $N D R G 4$ & $3108-3116(5)$ & $-106.2 \div-108.3$ \\
\hline$A K A P 11$ & $6559-6579(11)$ & -106.2 & NDUFS2 & 1917- $1925(5)$ & $-106.2 \div-108.3$ \\
\hline ANKLE1 & $2219-2255(12)$ & -106.2 & NKTR & $5828-5838(6)$ & -106.2 \\
\hline ARHGAP12 & $4800-4814(8)$ & -106.2 & NOS1 & $5560-5568(5)$ & -106.2 \\
\hline ATP9A & $3509-3523(8)$ & -106.2 & $O A S 3$ & $6257-6267(6)$ & -106.2 \\
\hline$B A C H 1$ & $4266-4274(8)$ & $-106.2 \div-110.4$ & PAK6 & $3163-3179(9)$ & -106.2 \\
\hline$B A C H 2$ & $5578-5604(14)$ & -106.2 & PARN & 2839-2861 (12) & -106.2 \\
\hline$B A Z 2 A$ & $6856-6868(7)$ & -106.2 & $P C B D 2$ & $1805-1815(6)$ & -106.2 \\
\hline$B G N$ & 1960-1980(11) & -106.2 & PCK1 & $2388-2414(9)$ & $-106.2 \div-108.3$ \\
\hline C11orf75 & 693-709 (9) & $-106.2 \div-108.3$ & PDE1A & 2793-2805 (7) & $-106.2 \div-108.3$ \\
\hline C21orf91 & $5113-5121(5)$ & -106.2 & PDE3A & $4022-4040(10)$ & -106.2 \\
\hline C2orf91 & $1577-1593(7)$ & -106.2 & PEAK1 & $7268-7290(12)$ & $-106.2 \div-108.3$ \\
\hline$C A C F D 1$ & $2283-2303(9)$ & $-106.2 \div-108.3$ & $P I G S$ & $2585-2597(7)$ & -106.2 \\
\hline CACNG8 & $6807-6817(6)$ & -106.2 & $P K N O X 1$ & $1680-1688(7)$ & $-106.2 \div-108.3$ \\
\hline$C B X 3$ & $1306-1314(5)$ & $-106.2 \div-108.3$ & $P L A 2 G 7$ & $1643-1651(5)$ & $-106.2 \div-108.3$ \\
\hline$C C D C 9$ & $1815-1833(10)$ & -106.2 & PLEKHA2 & $1672-1684(7)$ & -106.2 \\
\hline$C D 2 A P$ & $2829-2849(11)$ & $-106.2 \div-108.3$ & $P R K X$ & 5366- $5390(13)$ & -106.2 \\
\hline$C D 36$ & $3530-3538(5)$ & $-106.2 \div-108.3$ & PTGES & $1664-1688(13)$ & -106.2 \\
\hline CDK6 & $1907-1917(8)$ & $-106.2 \div-108.3$ & PTP4A2 & $3572-3586(8)$ & $-106.2 \div-108.3$ \\
\hline CHRDL1 & $3452-3474(12)$ & -106.2 & PTPN3 & $7001-7039(20)$ & -106.2 \\
\hline CLASP1 & 6695-6711 (9) & -106.2 & QSOX2 & $3460-3476(9)$ & $-106.2 \div-108.3$ \\
\hline CNOT6 & $5094-5104(6)$ & $-106.2 \div-108.3$ & $R A B G A P 1$ & $4103-4105(7)$ & $-106.2 \div-108.3$ \\
\hline$D B T$ & 5929-5965 (19) & $-106.2 \div-108.3$ & RAPGEFL1 & $2305-2339(18)$ & -106.2 \\
\hline$D M X L 1$ & 9853-6922 (5) & $-106.2 \div-108.3$ & $R D X$ & $3321-3329(5)$ & -106.2 \\
\hline DONSON & $2372-2446(38)$ & -106.2 & REEP3 & $3836-3856(12)$ & $-106.2 \div-108.3$ \\
\hline ELOF1 & $429-455$ (14) & -106.2 & $R O R A$ & $5293-5307(8)$ & -106.2 \\
\hline ETS1 & 3887-3907 (11) & -106.2 & $S A M D 4 A$ & 4191-4201 (6) & -106.2 \\
\hline$E V I 2 A$ & $2015-2027(7)$ & -106.2 & SEPT3 & $1319-1333(8)$ & -106.2 \\
\hline
\end{tabular}


Continuation of table «c»

\begin{tabular}{|c|c|c|c|c|c|}
\hline Gene & $\begin{array}{l}\text { Start positions of } \\
\text { binding sites, nt }\end{array}$ & $\Delta \mathrm{G} / \Delta \mathrm{G}_{\mathrm{m}}, \mathrm{kJ} / \mathrm{mole}$ & Gene & $\begin{array}{l}\text { Start positions of } \\
\text { binding sites, } \mathrm{nt}\end{array}$ & $\Delta \mathrm{G} / \Delta \mathrm{G}_{\mathrm{m}}, \mathrm{kJ} / \mathrm{mole}$ \\
\hline FAM120C & $4206-4222(9)$ & -106.2 & SERBP1 & $5153-5163(6)$ & -106.2 \\
\hline FAM126B & $3744-3764$ (7) & $-106.2 \div-108.3$ & $S F N$ & $1189-1199(6)$ & -106.2 \\
\hline FAM180B & $928-940(7)$ & -106.2 & SH3PXD2A & $8615-8623(5)$ & -106.2 \\
\hline FAM212B & $1317-3453(7)$ & -106.2 & SLC1A5 & $2651-2679(10)$ & -106.2 \\
\hline FAM216B & $1390-1398(5)$ & -106.2 & $S L C 25 A 44$ & 1893-1913 (11) & -106.2 \\
\hline FASLG & $1603-1613(6)$ & $-106.2 \div-108.3$ & SLC $30 A 3$ & 1865-1905 (9) & -106.2 \\
\hline$F G F 9$ & $1737-1745(8)$ & $-106.2 \div-108.3$ & SLFN5 & $3046-3058(7)$ & $-106.2 \div-108.3$ \\
\hline FLT1 & $6910-6922(8)$ & $-106.2 \div-108.3$ & SMIM15 & $2574-2584(6)$ & -106.2 \\
\hline FOXK1 & $10612-10628(9)$ & $-106.2 \div-108.3$ & $S P 1$ & $4146-4158(7)$ & -106.2 \\
\hline$G G A 2$ & $2081-2103(12)$ & -106.2 & ST8SIA1 & $4536-4558(12)$ & -106.2 \\
\hline GID4 & $2279-2291(7)$ & $-106.2 \div-108.3$ & SYNPO2L & $3586-3600(8)$ & -106.2 \\
\hline GLCCI1 & $3955-3963(5)$ & $-106.2 \div-108.3$ & SYT1 & $2739-2749(6)$ & -106.2 \\
\hline GNAI1 & $2937-2945(5)$ & -106.2 & SYT2 & 1863-1907 (15) & $-106.2 \div-108.3$ \\
\hline GPR21 & $1481-1503(12)$ & $-106.2 \div-108.3$ & SYTL4 & $2462-2480(6)$ & $-106.2 \div-110.4$ \\
\hline GTPBP1 & $2471-2485(8)$ & -106.2 & $T B X 4$ & 1780-1798 (10) & $-106.2 \div-108.3$ \\
\hline$H E M G N$ & $1841-1849(6)$ & $-106.2 \div-108.3$ & $T I P R L$ & $2688-2702(8)$ & $-106.2 \div-108.3$ \\
\hline HPS4 & 3984-3998 (8) & -106.2 & TMEM132B & $5871-5879(5)$ & -106.2 \\
\hline$I G F 2 R$ & $8446-8454(5)$ & -106.2 & TMEM2 & $6337-6345(5)$ & -106.2 \\
\hline$J A K 2$ & 5183-5199(9) & -106.2 & TMEMЗОВ & $2800-2812(7)$ & -106.2 \\
\hline KCNJ10 & $3349-3375$ (14) & -106.2 & TNFRSF 21 & $2497-2517(7)$ & -106.2 \\
\hline KCNJ12 & 4656- $4670(8)$ & $-106.2 \div-108.3$ & TNIP3 & $1604-1620(9)$ & $-106.2 \div-108.3$ \\
\hline KCNK10 & $5523-5547(13)$ & $-106.2 \div-108.3$ & $U B N 2$ & 10914-10924 (6) & -106.2 \\
\hline KIAA2026 & 6628-6644 (9) & -106.2 & $U M P S$ & $4221-4241(10)$ & -106.2 \\
\hline KIFCl & $2488-2512(13)$ & -106.2 & $U N C 5 B$ & $4036-4046(6)$ & -106.2 \\
\hline LANCL3 & $2972-2980(5)$ & $-106.2 \div-108.3$ & $V A P B$ & $2523-2537(8)$ & $-106.2 \div-108.3$ \\
\hline LSM14A & $2048-2070(10)$ & $-106.2 \div-108.3$ & WDR3 & $3560-3588(11)$ & $-106.2 \div-110.4$ \\
\hline$M A C C 1$ & $3950-3966(10)$ & $-106.2 \div-108.3$ & $W T 1$ & $2704-2714(6)$ & -106.2 \\
\hline MARCH5 & 1863-1879 (9) & $-106.2 \div-108.3$ & $\mathrm{ZC} 3 \mathrm{H} 12 \mathrm{C}$ & $6510-6528(10)$ & -106.2 \\
\hline MCTS1 & $1294-1318(7)$ & $-106.2 \div-108.3$ & ZDHHC21 & $7360-7374(8)$ & -106.2 \\
\hline MGAT5 & $4307-4333(14)$ & $-106.2 \div-108.3$ & ZNF670 & $1930-1940(7)$ & $-106.2 \div-108.3$ \\
\hline MLLT4 & $7131-7153(12)$ & -106.2 & $Z X D A$ & $3942-3952(6)$ & $-106.2 \div-108.3$ \\
\hline
\end{tabular}

The binding characteristics of miR-466-3p in the 3'UTR of 147 target genes mRNAs with the value $\Delta \mathrm{G} / \Delta \mathrm{Gm}$ equal or greater than $90 \%$ are shown in Table «b». The number of miR-466-3p binding sites on mRNAs of these genes varied from one to four, and indicates the different probability of miR-466-3p interaction with mRNAs, since the greater the length of the section of longer binding sites, is associated with a higher the probability of interaction. ZNF family transcription factors are among of target genes, and they have less than four binding sites. Only ZNF670 gene mRNA has seven binding sites (Table $\langle\mathrm{c}\rangle)$. miR-466-3p target genes involved in many biological processes and, therefore, miR-466-3p may participate in the regulation and can cause diseases. Table «d» demonstrates some of these genes.

Table «c» demonstrates characteristics of miR-466-3p binding in the 3'UTR of target genes mRNAs, having five or more binding sites in the mRNA. 
Table «d»-miR-466-3p target genes involved in many biological processes

\begin{tabular}{|c|c|}
\hline Functional group & Genes \\
\hline $\begin{array}{l}\text { Transcription } \\
\text { factors }\end{array}$ & 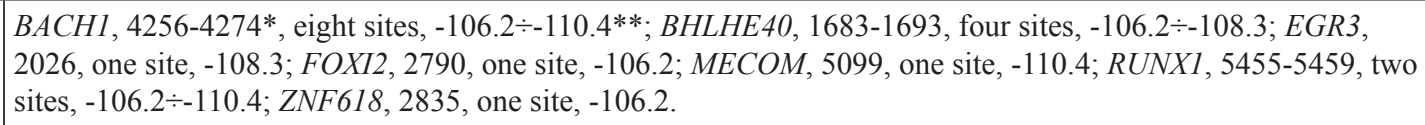 \\
\hline Kinases & 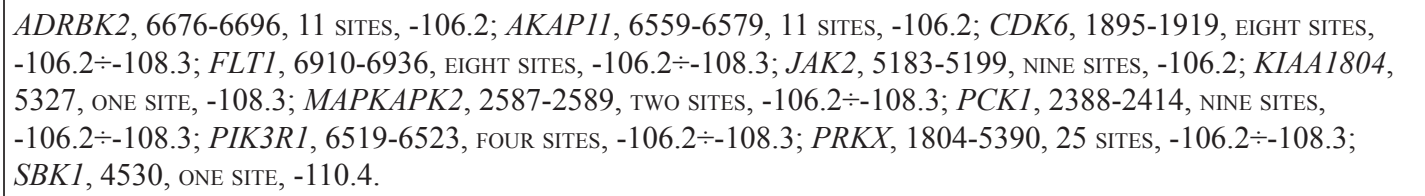 \\
\hline $\begin{array}{l}\text { Genes of } \\
\text { cell cycle }\end{array}$ & $\begin{array}{l}\text { BACH1; BHLHE40; CBX3, 1306-1314, five sites, }-106.2 \div-108.3 ; C D K 6 ; D D B 1,3990-4004, \text { three sites, } \\
-106.2 \div-108.3 ; \text { MECOM; WDR3, 3560-3588, } 11 \text { sites, }-106.2 \div-110.4 ; \text { MACC1, 2979-3966, } 10 \text { sites, }-106.2 \div- \\
108.3 ; \text { MAPKAPK2; NDRG4, 3108-3116, five sites, }-106.2 \div-108.3 ; \text { PIK3R1. }\end{array}$ \\
\hline $\begin{array}{l}\text { Genes of } \\
\text { apoptosis }\end{array}$ & 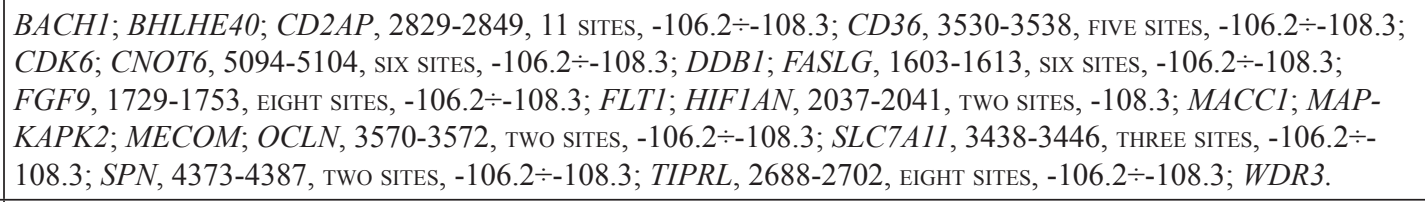 \\
\hline $\begin{array}{l}\text { Genes of breast } \\
\text { cancer }\end{array}$ & 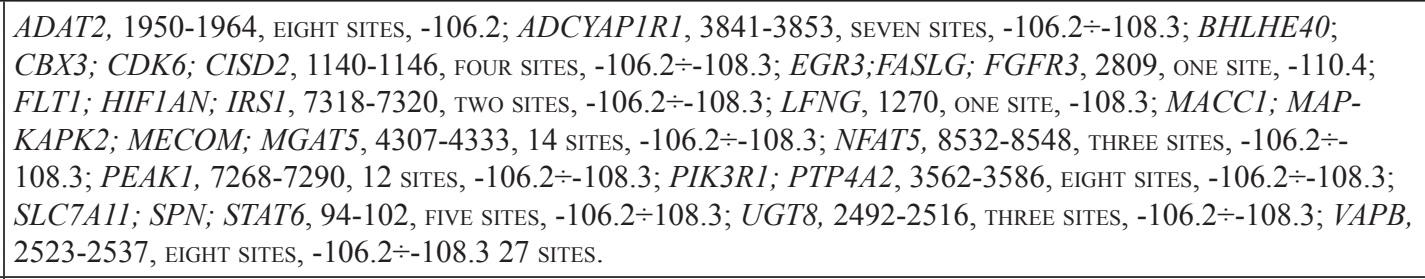 \\
\hline $\begin{array}{l}\text { Genes of lung } \\
\text { cancer }\end{array}$ & $\begin{array}{l}\text { ADAT2; ADCYAP1R1; BHLHE40; CDK6; DPYSL5, 4857, ONE SITE, -106.2; FASLG; FGF9; FGFR3; FLT1; } \\
\text { HEMGN, 1841-1849, SIX SITES, -106.2 } \div-108.3 ; \text { MACC1; MAPKAPK2; MECOM; LILRB2, 2858-2864, FOUR } \\
\text { SITES, -106.2 } \div-108.3 ; \text { SPN; STAT6; UGT8 } 17 \text { SITES. }\end{array}$ \\
\hline $\begin{array}{l}\text { Genes of } \\
\text { cardiovas-cular } \\
\text { diseases }\end{array}$ & 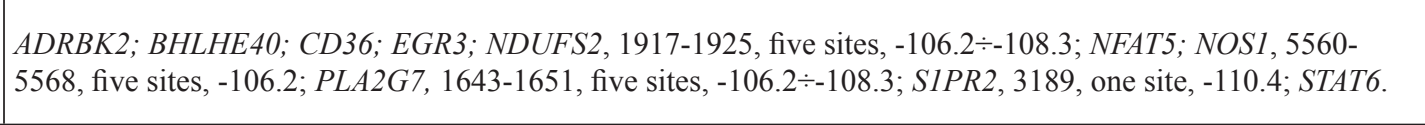 \\
\hline $\begin{array}{l}\text { Genes of } \\
\text { circadian rhythm }\end{array}$ & BHLHE40; DBT, 5929-5965, 19 sites, $-106.2 \div-108.3 ;$ NR1D2, 3337, one site, -110.4 . \\
\hline
\end{tabular}

Typically, the more multiple binding sites available in the mRNA, the more extended is the section. mRNA of LSM14A and PARN genes having 10 and 12 binding sites, have a length of 45 nucleotides. mRNA of $B A C H 2, K C N J 10$ genes containing 10 and 12 binding sites, have a length of 49 nt. mRNA of RAPGEFL1, PTPN3 genes containing 18 and 21 binding sites, have a length of $57 \mathrm{nt}$ and $61 \mathrm{nt}$, respectively. mRNA of gene containing 38 binding sites, have a length $97 \mathrm{nt}$. mRNAs of the above genes can bind more than one RISC complex containing miR-466-3p and efficiency of translation of mRNA suppression will be higher. We have developed a program of predicting miRNA binding sites with mRNAs which allows high reliability to establish these sites. At the value of $\Delta \mathrm{G} / \Delta \mathrm{Gm}$ equal to $90 \%$, the level of $p<0.0001$. For example, we were able to identify 200 genes mRNAs from 18,000 human genes which have entirely complementary binding sites for unique miR-619.

Considering the possibility of regulating that the expression of many genes are regulated by miR-466$3 p$, it should be expected that the concentration of miR-466-3p should notis not expected to change in the norm vary widely; otherwise, excessively high or low levels of miR-466-3p would inevitably lead to the disruption of the expression of genes involved in key biological processes and would result in several pathological conditions. The type of disease will depends on the ratio of the concentrations of miR-466$3 p$ and target mRNAs of target genes in specific cells and tissues of the body [12-18]. 


\section{References}

1. Niyazova R., Berillo O., Atambayeva Sh., Pyrkova A., Alybaeva A., Ivashchenko A. miR-1322 Binding Sites in Paralogous and Orthologous Genes. // Biomed Research International. - 2015. - P. 1-7.

2. Ivashchenko A., Berillo O., Pyrkova A., Niyazova R., Atambayeva Sh. Binding sites of mir1273 family on the mRNA of target genes. // Biomed research international. -2014 . - P. 1-8.

3. Ivashchenko A., Berillo O., Pyrkova A., Niyazova R., Atambayeva Sh. The properties of binding sites of miR-619-5p, miR-5095, miR-5096 and miR$5585-3 p$ in the mRNAs of human genes. // Biomed research international. - 2014. - P. 2014.

4. Ivashchenko A., Berillo O., Pyrkova A., Niyazova R., Atambayeva Sh. MiR-3960 binding sites with mRNA of human genes. // Bioinformation. 2014. - Vol. 10. - No. 7. - P. 423-427.

5. Cheng Q., Yi B., Wang A., Jiang X. Exploring and exploiting the fundamental role of microRNAs in tumor pathogenesis. // Onco Targets Ther. 2013. - Vol. 6. - P. 1675-1684.

6. Hromadnikova I., Kotlabova K., Hympanova L., Krofta L. Cardiovascular and cerebrovascular desease associated microRNAs are dysregulated in placental tissues affected with gestational hypertension, preeclampsia and intrauterine growth restriction. // Plos One. - 2015. - Vol. 10 - No. 9. - e0138383.

7. Faruq O., Vecchione A. microRNA: Diagnostic perspective. // Front Med. - 2015. - 2. - P. 51.

8. Kool E.T. Hydrogen bonding, base stacking, and steric effects in DNA replication. // Annu.Rev. Biophys.Biomol.Struct. - 2001. - Vol. 30. - P. 1-22.

9. Leontis N.B., Stombaugh J., Westhof E. The non-Watson-Crick base pairs and their associated isostericity matrices. // Nucleic Acids Res. - 2002. Vol. 30. - P. 3497-3531.

10. Ivashchenko A., Berillo O., Pyrkova A., Niyazova R., Atambayeva S. MiR-3960 binding sites with mRNA of human genes. // Bioinformation. 2014. - Vol. 10. - No. 7. - P. 423-427.

11. Berillo O., Atambayeva Sh., Niyazova R., Akimniyazova A., Ivashchenko A., Pyrkova A. Features of Multiple MiR-574-5p Binding Sites and Functions of Their Target-Genes. // Journal of Bioinformatics and Computational Biology. - submitted for publication.

12. Druz A., Chu C., Majors B., Santuary R., Betenbaugh M., Shiloach J. A novel microRNA mmu-miR-466h affects apoptosis regulation in mammalian cells. // Biotechnol Bioeng. - 2011. Vol. 108. - P. 1651-1661.

13. Dimmeler S., Zeiher A.M. Endothelial cell apoptosis in angiogenesis and vessel regression. // Circ Res. - 2000. - Vol. 87. - P. 434-439.

14. Folkman J. Angiogenesis and apoptosis. // Semin Cancer Biol. - 2003. - 13. - P. 159-167.

15. Druz A., Betenbaugh M., Shiloach J. Glucose depletion activates mmu-miR-466h-5p expression through oxidative stress and inhibition of histone deacetylation. // Nucleic Acids Res. - 2012. - Vol. 40. - P. 7291-7302.

16. Li Y., Fan X., He X., Sun H., Zou Z., Yuan H., Xu H., Wang C., Shi X. MicroRNA-466 inhibits antiviral innate immune response by targeting interferon-alpha. // Cell Mol Immunol. - 2012. - Vol. 9. - P. 497-502.

17. Luo Y., Liu Y., Liu M., Wei J., Zhang Y., Hou J., Huang W., Wang T., Li X., He Y., Ding F., Yuan L., Cai J., Zheng F., Yang J. Sfmbt2 10th intron-hosted miR-466(a/e)-3p are important epigenetic regulators of Nfat5 signaling, osmoregulation and urine concentration in mice. // Biochim Biophys Acta. - 2014. - Vol. 1839. - P. 97-106.

18. Seo M., Choi J., Rae Rho Ch., Joo Ch., Lee S. Microrna Mir-466 inhibits lymphangiogenesis by targeting Prospero-related homeobox 1 in the alkali burn corneal injury model. // Journal of Biomedical Science. - 2015. - Vol. 22. - P. 3. 